\title{
Algebraic structure of the perfect Ree-Tits generalized octagons
}

\author{
Kris Coolsaet
}

\begin{abstract}
We provide an algebraic description of the perfect Ree-Tits generalized octagons, i.e., an explicit embedding of octagons of this type in a 25-dimensional projective space. The construction is derived from the interplay between the 52-dimensional module of the Chevalley algebra of type $\mathrm{F}_{4}$ over a field of even characteristic and its 26-dimensional submodule. We define a quadratic duality operator that interchanges special sets of (totally) isotropic elements in those modules and establish the points of the octagon as absolute points of this duality. We introduce many algebraic operations that can be used in the study of the generalized octagon. We also prove that the Ree group acts as expected on points and pairs of points.
\end{abstract}

Keywords: Ree-Tits generalized octagon, Chevalley algebra of type F4

MSC 2000: 51E12, 17B25

\section{Contents}

1 Introduction and overview . . . . . . . . . . . 2

2 The Chevalley algebra of type $\mathrm{F}_{4} \ldots \ldots \ldots 5$

3 Isotropic and totally isotropic elements . . . . . . . . 18

4 Automorphisms .................... 23

5 The quotient algebra $\mathrm{Q} \ldots \ldots \ldots 31$

6 The operator $Q(\cdot) \ldots \ldots \ldots 33$

7 Octagonality ................... 41

8 The Ree group ${ }^{2} \widehat{\mathrm{F}_{4}}(K) \ldots \ldots \ldots \ldots$. . . . . . . . . 43

9 Pairs of octagonal elements . . . . . . . . . . . . . 50

10 The Ree-Tits octagon . . . . . . . . . . . . . 56 


\section{Introduction and overview}

Of all generalized polygons related to simple groups [5] the generalized octagons have probably been studied the least. Perhaps one of the reasons is the fact that only a single (infinite) family of examples is known and that these Ree-Tits octagons, and their embeddings in a projective space, are not easily constructed.

The standard way to define a Ree-Tits octagon is as a coset geometry of a Ree group. This group is itself constructed by 'twisting' the Chevalley group of type $\mathrm{F}_{4}$ over a suitable field $K$ of characteristic 2 . Another construction technique makes use of a special coordinatization (introduced by H. Van Maldeghem [5]) which is itself based on the properties of the Ree groups, in particular the commutation relations displayed in [4].

What is missing for the Ree-Tits octagon, and exists for all other classical generalized polygons, is an explicit embedding into some projective?space. In Section 10 of this text we shall construct such an embedding into a 25-dimensional space, where the points (lines) of the Ree-Tits octagon are a subset of the points (lines) of the projective space. We will provide explicit 'formulae' which can be applied to the projective coordinates of a point to determine whether or not it is a point of the octagon. And likewise, we shall provide a means to determine from the projective coordinates of two points of the octagon, whether they are collinear in the octagon, and more generally, what is their mutual distance.

These 'formulae' are not so elementary as in the case of the other generalized polygons. For example, we shall prove that a point $K e$ belongs to the Ree-Tits octagon if and only if both $e^{2}=0$ and $q([e, \mathbf{W}], e)=0$. The first expression is shorthand for a system of 26 quadratic equations in 26 variables (the 26 coordinates of $e$ ). The second expression corresponds to a system of 676 polynomials of degree 3 in 26 variables, additionally involving a field automorphism $\sigma$ of $K$. (For contrast, compare this to the formulae needed to define the points of the split Cayley hexagon : a single quadratic equation in 7 variables suffices.)

This text looks at the octagon (and the metasymplectic space to which its points and lines belong) from three different perspectives : groups, geometries and algebras, concentrating on the latter.

We start with the well-known 52-dimensional Chevalley algebra $\mathbf{F}$ of type $\mathrm{F}_{4}$ and its 26-dimensional module $\mathrm{W}$ in the special case of characteristic 2. In this particular case $\mathbf{W}$ is a subalgebra of $\mathbf{F}$. (Many of the definitions and propositions in the first few sections of this text can also be extended to fields with different characteristics.)

$\mathbf{W}$ is isomorphic to the exceptional (quadratic) 26-dimensional Jordan alge- 




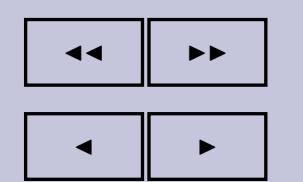

page $6 / 65$

go back

full screen

close

quit

The value of $\langle r, s\rangle$ is closely related to whether $r+s, r-s$ are roots and of what kind, as summarised in the following tables :

When $r, s \in \Phi_{S}$

\begin{tabular}{|c|l|}
\hline$\langle r, s\rangle=$ & if and only if \\
\hline 2 & $r=s$ \\
1 & $r-s \in \Phi_{S}$ \\
0 & both $r-s, r+s \in \Phi_{L}$ \\
-1 & $r+s \in \Phi_{S}$ \\
-2 & $r=-s$ \\
\hline
\end{tabular}

When $r \in \Phi_{S}, s \in \Phi_{L}$

\begin{tabular}{|c|l|}
\hline$\langle r, s\rangle=$ & if and only if \\
\hline 2 & $r-s \in \Phi_{S}$ \\
0 & $r-s, r+s \notin \Phi$ \\
-2 & $r+s \in \Phi_{S}$ \\
\hline
\end{tabular}

When $r, s \in \Phi_{L}$

\begin{tabular}{|c|l|}
\hline$\langle r, s\rangle=$ & if and only if \\
\hline 2 & $r=s$ \\
1 & $r-s \in \Phi_{L}$ \\
0 & $r-s, r+s \notin \Phi$ \\
-1 & $r+s \in \Phi_{L}$ \\
-2 & $r=-s$ \\
\hline
\end{tabular}

When $r \in \Phi_{L}, s \in \Phi_{S}$

\begin{tabular}{|c|l|}
\hline$\langle r, s\rangle=$ & if and only if \\
\hline 1 & $r-s \in \Phi_{S}$ \\
0 & $r-s, r+s \notin \Phi$ \\
-1 & $r+s \in \Phi_{S}$ \\
\hline
\end{tabular}

With the root system $\Phi$ we may associate the dual root system $\Phi^{*}$ of roots $r^{*}$ of the form

$$
r^{*} \stackrel{\text { def }}{=} \frac{2 r}{r \cdot r}, \quad \text { with } r \in \Phi .
$$

The element $r^{*}$ is called the co-root corresponding to $r$. Note that $\left\langle r^{*}, s^{*}\right\rangle=$ $\langle s, r\rangle$.

The roots

$$
r_{1} \stackrel{\text { def }}{=} 1 \overline{1} 00, \quad r_{2} \stackrel{\text { def }}{=} 01 \overline{1} 0, \quad r_{3} \stackrel{\text { def }}{=} 0010, \quad r_{4} \stackrel{\text { def }}{=}---+,
$$

called simple roots, form a so-called fundamental system. Every root of $\Phi$ can be written as a linear combination of simple roots, with integral coefficients. If $r=\alpha_{1} r_{1}+\alpha_{2} r_{2}+\alpha_{3} r_{3}+\alpha_{4} r_{4}$, then

$$
\begin{aligned}
& \alpha_{1}=2\left\langle r_{1}, r\right\rangle+3\left\langle r_{2}, r\right\rangle+2\left\langle r_{3}, r\right\rangle+1\left\langle r_{4}, r\right\rangle \\
& \alpha_{2}=3\left\langle r_{1}, r\right\rangle+6\left\langle r_{2}, r\right\rangle+4\left\langle r_{3}, r\right\rangle+2\left\langle r_{4}, r\right\rangle \\
& \alpha_{3}=4\left\langle r_{1}, r\right\rangle+8\left\langle r_{2}, r\right\rangle+6\left\langle r_{3}, r\right\rangle+3\left\langle r_{4}, r\right\rangle \\
& \alpha_{4}=2\left\langle r_{1}, r\right\rangle+4\left\langle r_{2}, r\right\rangle+3\left\langle r_{3}, r\right\rangle+2\left\langle r_{4}, r\right\rangle
\end{aligned}
$$

With these notations the co-roots satisfy

$$
r^{*}=\left\{\begin{aligned}
2 \alpha_{1} r_{1}^{*}+2 \alpha_{2} r_{2}^{*}+\alpha_{3} r_{3}^{*}+\alpha_{4} r_{4}^{*}, & \text { when } r \in \Phi_{S}, \\
\alpha_{1} r_{1}^{*}+\alpha_{2} r_{2}^{*}+\frac{1}{2} \alpha_{3} r_{3}^{*}+\frac{1}{2} \alpha_{4} r_{4}^{*}, & \text { when } r \in \Phi_{L} .
\end{aligned}\right.
$$




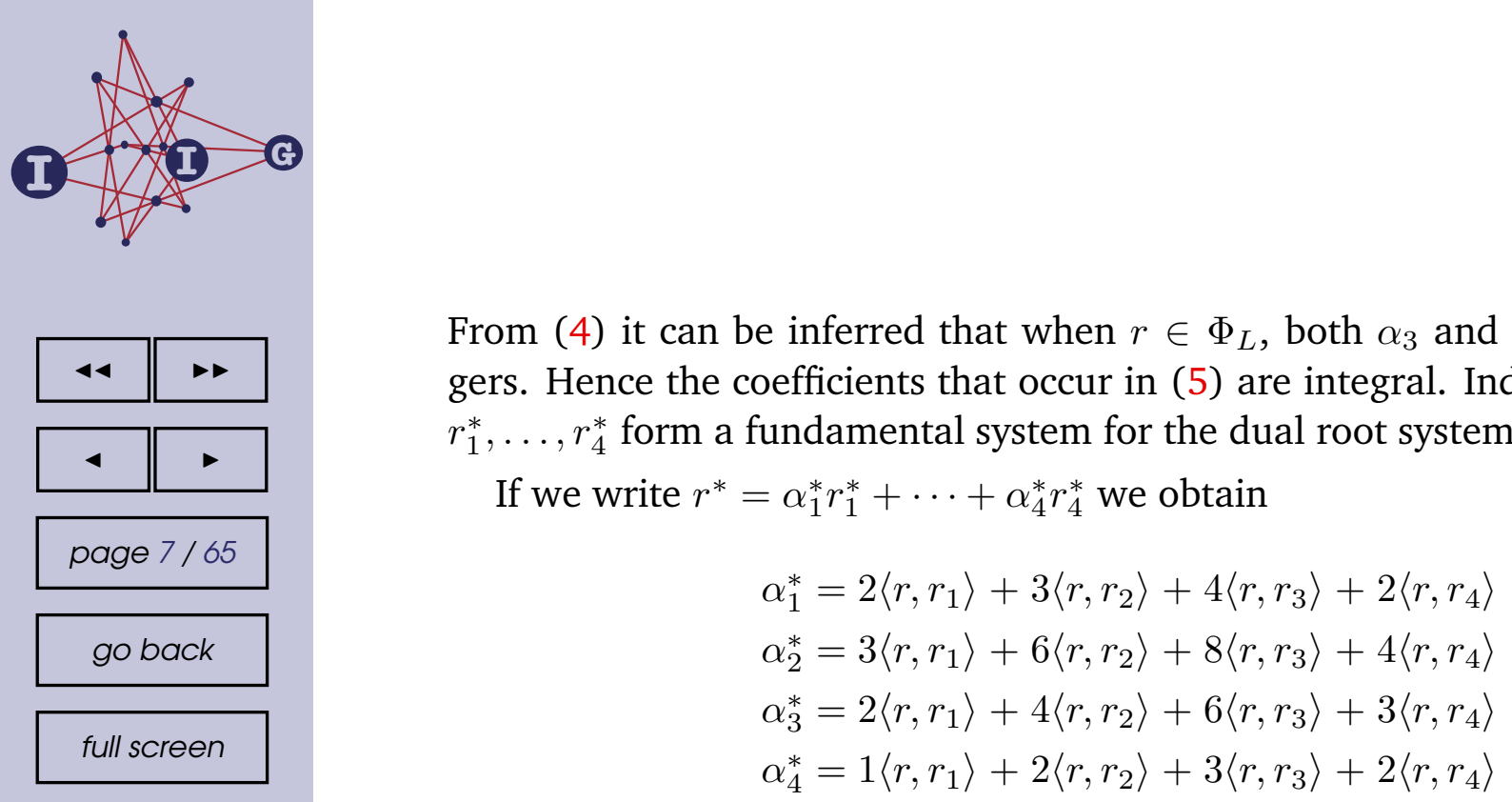

From (4) it can be inferred that when $r \in \Phi_{L}$, both $\alpha_{3}$ and $\alpha_{4}$ are even integers. Hence the coefficients that occur in (5) are integral. Indeed, the co-roots ${ }_{1}^{*}, \ldots, r_{4}^{*}$ form a fundamental system for the dual root system $\Phi^{*}$.

If we write $r^{*}=\alpha_{1}^{*} r_{1}^{*}+\cdots+\alpha_{4}^{*} r_{4}^{*}$ we obtain

$$
\begin{aligned}
& \alpha_{1}^{*}=2\left\langle r, r_{1}\right\rangle+3\left\langle r, r_{2}\right\rangle+4\left\langle r, r_{3}\right\rangle+2\left\langle r, r_{4}\right\rangle \\
& \alpha_{2}^{*}=3\left\langle r, r_{1}\right\rangle+6\left\langle r, r_{2}\right\rangle+8\left\langle r, r_{3}\right\rangle+4\left\langle r, r_{4}\right\rangle \\
& \alpha_{3}^{*}=2\left\langle r, r_{1}\right\rangle+4\left\langle r, r_{2}\right\rangle+6\left\langle r, r_{3}\right\rangle+3\left\langle r, r_{4}\right\rangle \\
& \alpha_{4}^{*}=1\left\langle r, r_{1}\right\rangle+2\left\langle r, r_{2}\right\rangle+3\left\langle r, r_{3}\right\rangle+2\left\langle r, r_{4}\right\rangle
\end{aligned}
$$

To every root $r$ we associate the reflection $w_{r}$ about the hyperplane orthogonal to $r$ :

$$
w_{r}(x) \stackrel{\text { def }}{=} x-\langle r, x\rangle r
$$

It is a (defining) property of a root system that these reflections always map roots onto roots. The finite group $W$ generated by all reflections $w_{r}$ with $r \in \Phi$ is the Weyl group of type $\mathrm{F}_{4}$. This group acts regularly on all fundamental systems of roots in $\Phi$ and it acts transitively on roots of the same type (short or long) and on pairs of roots with the same type and inner product. In other words, the 16 cases listed in (2) correspond exactly to the orbits of $W$ on ordered pairs $(r, s)$ of roots.

The Chevalley algebra $\mathbf{F}$ of type $\mathrm{F}_{4}$ over a field $K$ is a 52-dimensional vector space over $K$ with a bilinear 'Lie bracket' operator $[\cdot, \cdot]$. $\mathbf{F}$ can be written as a direct sum of the following form :

$$
\mathbf{F}=\mathbf{H} \oplus \bigoplus_{r \in \Phi} K e_{r}
$$

The elements $e_{r}$, one for every $r \in \Phi$, are the root vectors of $\mathbf{F}$, and $\mathbf{H}$ is the Cartan subalgebra of dimension 4 , generated by the elements $h_{r} \stackrel{\text { def }}{=}\left[e_{r}, e_{-r}\right]$ for $r \in \Phi$.

Not all elements $h_{r}, r \in \Phi$ are linearly independent. In fact, the elements $h_{r}$ can be expressed as linear combinations of the 4 elements $h_{r_{1}}, \ldots, h_{r_{4}}$, in a manner very similar to (5) :

$$
h_{r}=\left\{\begin{aligned}
2 \alpha_{1} h_{r_{1}}+2 \alpha_{2} h_{r_{2}}+\alpha_{3} h_{r_{3}}+\alpha_{4} h_{r_{4}}, & \text { when } r \in \Phi_{S}, \\
\alpha_{1} h_{r_{1}}+\alpha_{2} h_{r_{2}}+\frac{1}{2} \alpha_{3} h_{r_{3}}+\frac{1}{2} \alpha_{4} h_{r_{4}}, & \text { when } r \in \Phi_{L} .
\end{aligned}\right.
$$

The coefficients should now be interpreted as elements of the base field $K$. (In our particular case of characteristic 2 we could therefore have left out the terms involving $2 \alpha_{1}$ and $2 \alpha_{2}$.) Note that, because the characteristic of $K$ is small, it may happen that $h_{r}=h_{r^{\prime}}$ although $r \neq r^{\prime}$. 


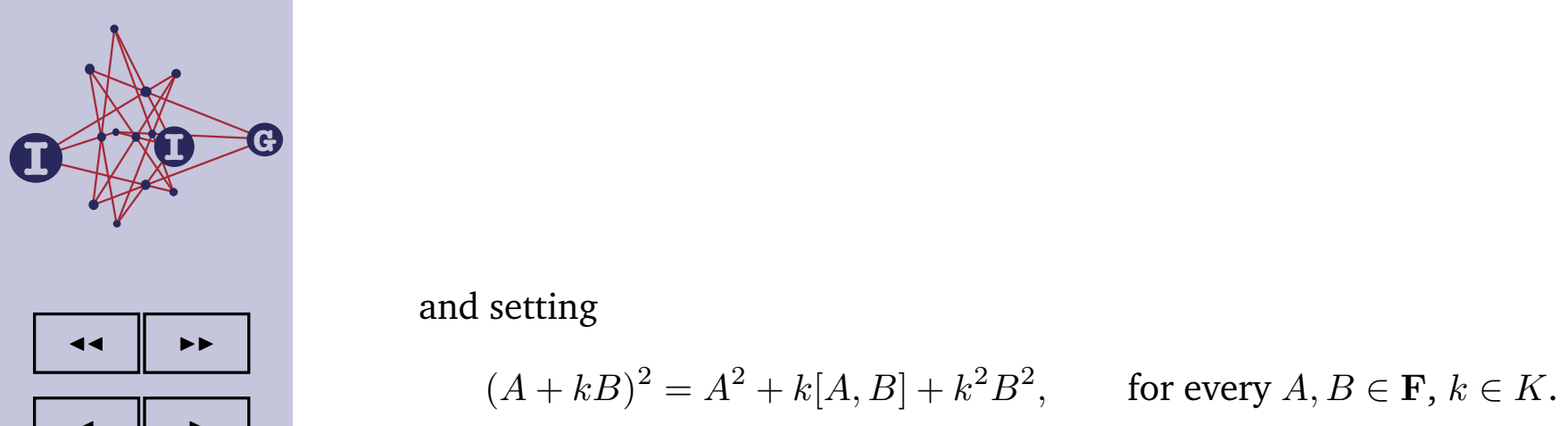

(Properties (12-13) uniquely determine the action of ${ }^{2}$ on F.)

In terms of coordinates we have :

$$
\begin{aligned}
& A^{2}[t]=\sum_{\substack{\{r, s\} \subset \Phi \\
r+s=t,\langle r, s\rangle \neq 0}} A[r] A[s]+\sum_{i=1}^{4}\left\langle r_{i}, t\right\rangle A[i] A[t], \quad \text { for } t \in \Phi, \\
& A^{2}[i]=A[i]^{2}+\sum_{\{r,-r\} \subset \Phi} A[r] A[-r] h_{r}[i], \quad \text { for } i=1, \ldots, 4 .
\end{aligned}
$$

(The first sum in the first equation treats each pair exactly once. The sum in the last equation consists of 24 terms, one for each pair $\{r,-r\}$ of roots. Equations (14) could serve as an alternative definition of.$^{2}$.)

Note that $h_{r}^{2}=h_{r}$ for all $r \in \Phi$ (and not only when $r=r_{i}$ is a simple root).

Proposition 2.1. Let $X, A \in \mathbf{F}$, then

$$
[[X, A], A]=\left[X, A^{2}\right] .
$$

Proof. The Jacobi identity proves that $[[X, A], B]+[[X, B], A]=[X,[A, B]]$, hence by (13) it is sufficient to prove (15) when $A$ is a Chevalley base element.

Let $r \in \Phi$. We express $\left[X, e_{r}\right]$ in terms of coordinates of $X$ :

$$
\left[X, e_{r}\right]=\sum_{\substack{s \in \Phi \\ r+s \in \Phi,\langle r, s\rangle \neq 0}} X[s] e_{r+s}+X[-r] h_{r}+\sum_{i} X[i]\left\langle r_{i}, r\right\rangle e_{r}
$$

We have $\langle r, r+s\rangle=\langle r, r\rangle+\langle r, s\rangle=2+\langle r, s\rangle \geq 0$, and hence $\left[e_{r+s}, e_{r}\right]=0$. Also $\left[h_{r}, e_{r}\right]=0$ and $\left[e_{r}, e_{r}\right]=0$, and therefore $\left[\left[X, e_{r}\right], e_{r}\right]=0=\left[X, e_{r}^{2}\right]$.

Similarly, $\left[X, h_{r}\right]=\sum_{s \in \Phi} X[s]\langle r, s\rangle e_{s}$, so $\left[\left[X, h_{r}\right], h_{r}\right]=\sum_{s \in \Phi} X[s]\langle r, s\rangle^{2} e_{s}$, which is the same as $\left[X, h_{r}\right]$ because $\langle r, s\rangle^{2}=\langle r, s\rangle$ in characteristic 2.

Let $\mathbf{W}$ denote the subspace of $\mathbf{F}$ generated by the elements $h_{r}, e_{r}$, restricted to the short roots $r \in \Phi_{S}$ :

$$
\mathbf{W} \stackrel{\text { def }}{=} \mathbf{I} \oplus \bigoplus_{r \in \Phi_{S}} K e_{r}
$$

where $\mathbf{I}$ is the subspace of $\mathbf{H}$ generated by all $h_{r}$ with $r \in \Phi_{S}$. In other words : $A \in \mathbf{W}$ if and only if its coordinates satisfy $A[r]=0$, for all $r \in \Phi_{L}$, and $A[1]=A[2]=0$. Hence $\mathbf{W}$ has dimension 26 . 


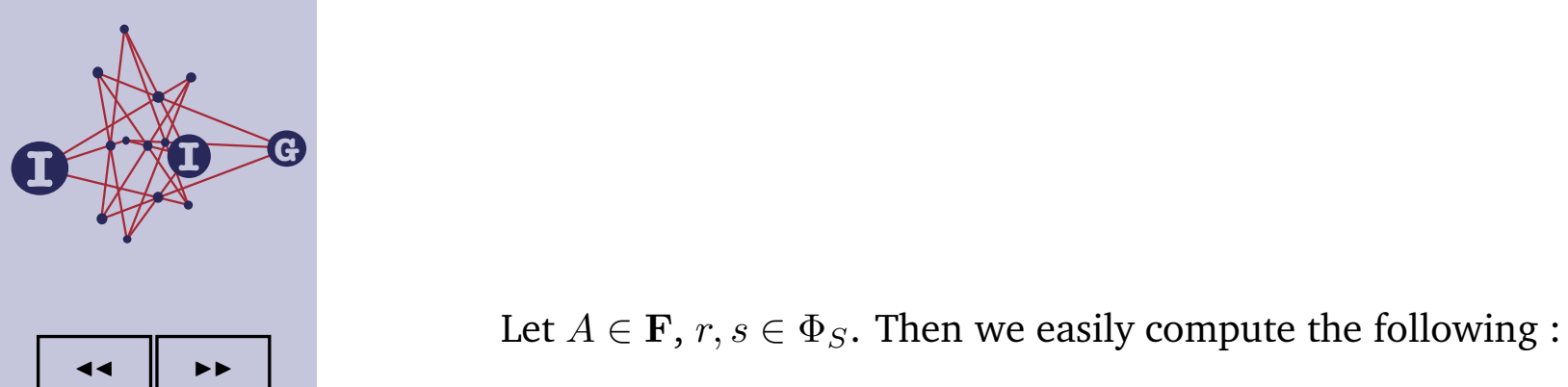

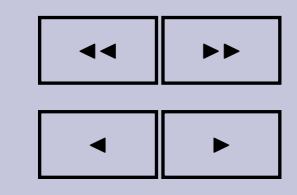

page $11 / 65$

go back

full screen

close

quit

$$
\begin{array}{ll}
{\left[e_{r}, A\right] \cdot e_{s}= \begin{cases}\sum_{i=1}^{4}\left\langle r_{i}, r\right\rangle A[i], & \text { when } r=-s, \\
A[-r-s], & \text { when }\langle r, s\rangle=0 \text { or }-1, \\
0, & \text { otherwise. }\end{cases} } \\
{\left[h_{s}, A\right] \cdot e_{r}=\langle s, r\rangle A[-r],} \\
{\left[e_{r}, A\right] \cdot h_{s}=\langle s, r\rangle A[-r],} \\
{\left[h_{r}, A\right] \cdot h_{s}=0 .}
\end{array}
$$

It follows that $[\mathbf{W}, A]=0$ for $A \in \mathbf{F}$ only when $A=0$.

As an immediate consequence of (20) we find

$$
[a, C] \cdot a=0, \quad[a, C] \cdot b=[b, C] \cdot a, \quad \text { for } a, b \in \mathbf{W} \text { and } C \in \mathbf{F} .
$$

When $C=c$ belongs to $\mathbf{W}$ we may interchange the roles of $a$ and $c$ in the above, to obtain

$$
[a, b] \cdot c=[b, c] \cdot a=[c, a] \cdot b, \quad \text { for all } a, b, c \in \mathbf{W} .
$$

Taking the dot product of $d$ with the Jacobi identity $[[a, b], c]+[[b, c], a]+[[c, a], b]=$ 0 , and applying (22) yields

$$
[a, b] \cdot[c, d]+[a, c] \cdot[b, d]+[a, d] \cdot[b, c]=0, \quad \text { for all } a, b, c, d \in \mathbf{W} .
$$

To the symmetric bilinear dot product we associate a quadratic norm function $N(\cdot)$ as follows :

$$
N\left(e_{r}\right) \stackrel{\text { def }}{=} 0, \quad N\left(h_{r_{i}}\right) \stackrel{\text { def }}{=} 1, \quad \text { for } r \in \Phi_{S}, i=3,4 .
$$

with

$$
N(a+k b)=N(a)+k a \cdot b+k^{2} N(b), \quad \text { for } a, b \in \mathbf{W}, k \in K .
$$

In terms of coordinates :

$$
N(a)=\sum_{\{r,-r\} \subset \Phi_{S}} a[r] a[-r]+a[3]^{2}+a[3] a[4]+a[4]^{2} .
$$

where the sum is taken such that every pair $\{r,-r\}$ occurs exactly once. Note that $N\left(h_{r}\right)=1$ for all $r \in \Phi_{S}$ (and not only when $r=r_{i}$ is a simple root).

The following is a basic structural 'axiom' for the algebra $\mathbf{W}$ :

Proposition 2.2. Let $a \in \mathbf{W}$, then

$$
\left(a^{2}\right)^{2}=N(a) a^{2}+\left(a^{2} \cdot a\right) a
$$




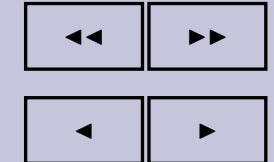

page $13 / 65$

go back

full screen

close

quit

written as $s_{0}+s_{i}, s_{0}+s_{i}+s_{j}, s_{0}+s_{i}+s_{j}+s_{k}$ or $s_{0}+s_{1}+s_{2}+s_{3}+s_{4}$, with $i, j, k \in\{1, \ldots, 4\}$. Also 1000 is the sum of +--- and ++++ and likewise for 0100, 0010 and 0001.

By (32) we may conclude that for every $r \in \Phi_{S}, H(r)$ can be written as a linear combination of $H\left(s_{0}\right), \ldots, H\left(s_{4}\right)$. However, these five values are not independent. Indeed, we have

$$
\begin{aligned}
& r \quad=--+=\left(\left(s_{0}+s_{1}\right)+s_{2}\right)+s_{3}, \\
& s \quad=++--=\left(\left(s_{0}+s_{3}\right)+s_{4}\right), \\
& r+s=00 \overline{1} 0=s_{3},
\end{aligned}
$$

and hence $H(r)=H\left(s_{0}\right)+H\left(s_{1}\right)+H\left(s_{2}\right)+H\left(s_{3}\right), H(s)=H\left(s_{0}\right)+H\left(s_{3}\right)+$ $H\left(s_{4}\right)$, and $H(r+s)=H\left(s_{3}\right)$. Therefore (32) implies $H\left(s_{1}\right)+H\left(s_{2}\right)+H\left(s_{3}\right)+$ $H\left(s_{4}\right)=0$.

We have

$$
\begin{array}{rlrrr}
s_{0}= & r_{1} & +2 r_{2} & +3 r_{3} & +r_{4} \\
s_{1}=-r_{1} & -r_{2} & -r_{3} & \\
s_{2}= & -r_{2} & -r_{3} & \\
s_{3}= & & -r_{3} & \\
s_{4}=-r_{1} & -2 r_{2} & -3 r_{3} & -2 r_{4} .
\end{array}
$$

Setting

$H_{1} \stackrel{\text { def }}{=} H\left(s_{2}\right)+H\left(s_{3}\right), H_{2} \stackrel{\text { def }}{=} H\left(s_{1}\right)+H\left(s_{2}\right), H_{3} \stackrel{\text { def }}{=} H\left(s_{0}\right)+H\left(s_{4}\right), H_{4} \stackrel{\text { def }}{=} H\left(s_{3}\right)$, we also easily verify that

$$
\begin{array}{lrrr}
H\left(s_{0}\right)=H_{2} & & +H_{4} & +H_{3} \\
H\left(s_{1}\right)=H_{2} & +H_{1} & +H_{4} & \\
H\left(s_{2}\right)= & H_{1} & +H_{4} \\
H\left(s_{3}\right)= & & H_{4} \\
H\left(s_{4}\right)=H_{2} & & +H_{4} . &
\end{array}
$$

Comparing (34) and (35) and using the fact that $\left\langle r_{i}, r_{j}\right\rangle$ is even unless $\{i, j\}=$ $\{1,2\}$ or $\{3,4\}$, we see that (33) is satisfied for $r=s_{0}, \ldots, s_{4}$. The lemma then follows because (33) is linear in $r$.

Proposition 2.4. Let $\tau \in \operatorname{Hom}(\mathbf{W}, \mathbf{W})$ be a linear transformation that satisfies

$$
\tau\left(a^{2}\right)=[\tau(a), a], \quad \text { for all } a \in \mathbf{W},
$$

or equivalently

$$
\tau\left(a^{2}\right) \cdot b=\tau(a) \cdot[a, b], \quad \text { for all } a, b \in \mathbf{W},
$$

then there exists a unique element $T \in \mathbf{F}$ such that $\tau(a)=[a, T]$ for every $a \in \mathbf{W}$. 








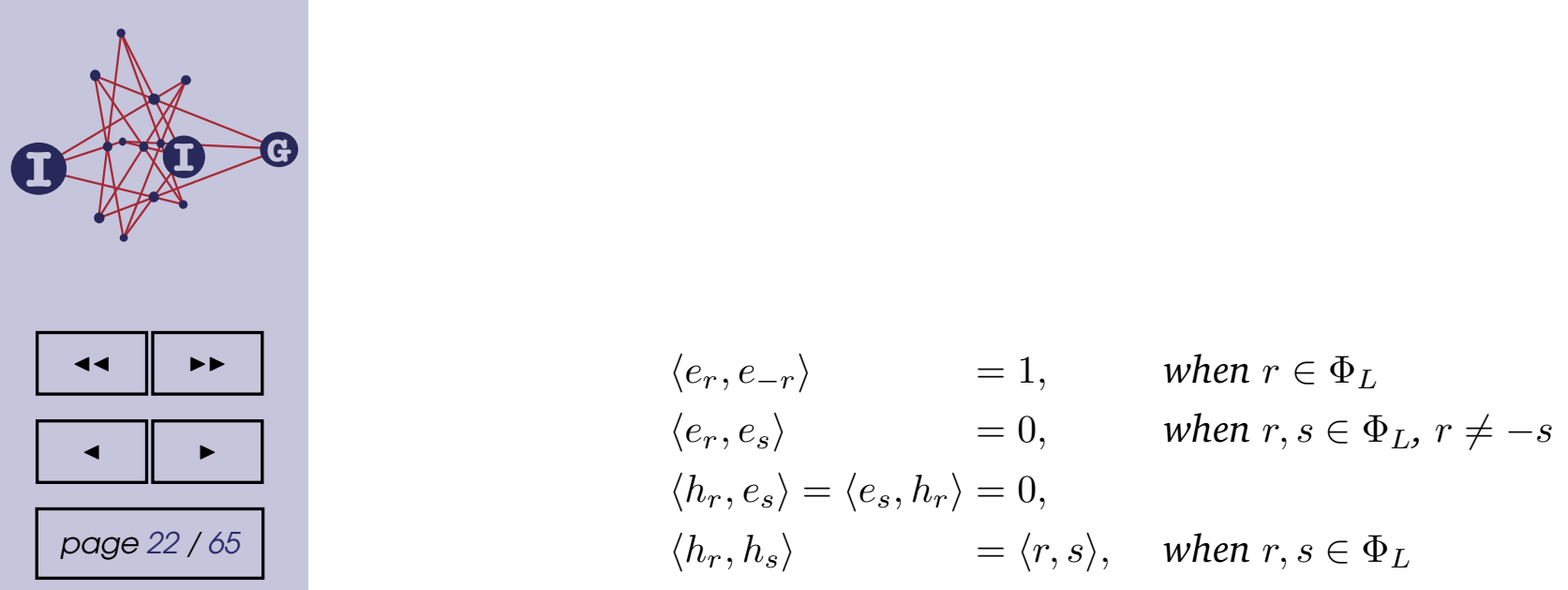

Proof. Note that by definition $\langle E, A\rangle$ is linear in its second argument. We need to prove that it can be 'made' linear in its first argument : in other words, if a linear combination $\sum_{i} k_{i} E_{i}$ of totally isotropic elements $E_{i}$ turns out to be zero, then $\sum_{i} k_{i}\left\langle E_{i}, A\right\rangle=0$ should be zero for every $A \in \mathbf{F}$.

Because $e_{r}$ and $e_{r}+e_{-r}+h_{r}$ are totally isotropic for every $r \in \Phi_{L}, A$ can always be written as a linear combination of totally isotropic elements and elements of $\mathbf{W}$. Hence it is sufficient to prove the linearization property above in the case that $A$ is totally isotropic and in the case that $A$ belongs to $\mathbf{W}$.

When $A$ is totally isotropic, we claim that $\langle E, A\rangle=\langle A, E\rangle$. Indeed, let $\{e, f\}$ be a defining pair for $E$ and $\left\{e^{\prime}, f^{\prime}\right\}$ for $A$, then

$$
\begin{aligned}
\langle E, A\rangle=\left[e, e^{\prime} * f^{\prime}\right] \cdot f & =\left[\left[e, e^{\prime}\right], f^{\prime}\right] \cdot f+\left(e \cdot f^{\prime}\right)\left(e^{\prime} \cdot f\right)+\left(e \cdot e^{\prime}\right)\left(f \cdot f^{\prime}\right) \\
& =\left[e, e^{\prime}\right] \cdot\left[f, f^{\prime}\right]+\left(e \cdot f^{\prime}\right)\left(e^{\prime} \cdot f\right)+\left(e \cdot e^{\prime}\right)\left(f \cdot f^{\prime}\right),
\end{aligned}
$$

and this identity remains unchanged when interchanging $e$ with $e^{\prime}$ and $f$ with $f^{\prime}$.

So in this case,

$$
\sum_{i} k_{i}\left\langle E_{i}, A\right\rangle=\sum_{i} k_{i}\left\langle A, E_{i}\right\rangle=\left\langle A, \sum_{i} k_{i} E_{i}\right\rangle=0
$$

as $\langle\cdot, \cdot\rangle$ is known to be linear in its second argument.

When $A$ belongs to $\mathbf{W}$, we have $\langle E, A\rangle=[e, A] \cdot f=[e, f] \cdot A=0$, by (21). Hence $\sum_{i} k_{i}\left\langle E_{i}, A\right\rangle$ is trivially zero.

This proves that $\langle\cdot, \cdot\rangle$ can be extended to a bilinear operator that is fully defined over F. We shall now establish that the formulae (50) and (51) are the only ones possible. Because every element of $\mathbf{F}$ can be written as a linear combination of an element of $\mathbf{W}$ and elements of the form $e_{r}, h_{r}$, with $r \in \Phi_{L}$, this will prove that the bilinear product is unique.

Consider the special case $E=e_{r}$ with $r \in \Phi_{L}$. By (45) we may write $e_{r}=$ $e_{s} * e_{t}$ with $s, t \in \Phi_{S}$ such that $s+t=r$. By (49) and (20) we have $\left\langle e_{r}, A\right\rangle=$ $\left[e_{s}, A\right] \cdot e_{t}=A[-s-t]=A[-r]$. This proves $\left\langle e_{r}, h_{s}\right\rangle=0$ and $\left\langle e_{r}, e_{s}\right\rangle=0$ for every $s \in \Phi$, except the case $\left\langle e_{r}, e_{-r}\right\rangle=1$. 






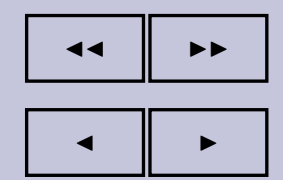

page $27 / 65$

go back

full screen

close

quit

Lemma 4.5. Let $e$ be an isotropic element of $\mathbf{W}, E$ a totally isotropic element of $\mathbf{F}$ and $g$ an automorphism of $\mathbf{F}$. Then

$$
x(e)^{g}=x\left(e^{g}\right), \quad x(E)^{g}=x\left(E^{g}\right) .
$$

Proof. Let $A \in \mathbf{F}$. We have

$$
\left(A^{x(e)}\right)^{g}=(A+[e, A]+[e, A] * e)^{g}=A^{g}+\left[e^{g}, A^{g}\right]+\left[e^{g}, A^{g}\right] * e^{g}=\left(A^{g}\right)^{x\left(e^{g}\right)},
$$

hence $x(e) g=g x\left(e^{g}\right)$. The second equation is proved in a similar way.

Proposition 4.6. Let $e, f$ be isotropic elements of $\mathbf{W}$ such that $e \cdot f=0$. Let $E, F$ be totally isotropic elements of $\mathbf{F}$ such that $\langle E, F\rangle=0$. Then

$$
\begin{aligned}
& x(e) x(f)=x(f) x(e) x([e, f]), \\
& x(e) x(E)=x(E) x(e) x([e, E]) x(e *[e, E]), \\
& x(E) x(F)=x(F) x(E) x([E, F]) .
\end{aligned}
$$

If moreover $[e, f]=0$ then

$$
x(e * f)=x(e) x(f) x(e+f) .
$$

Proof. First consider the case $[e, f]=0$. Note that in that case $f^{x(e)}=f+[e, f]+$ $(e \cdot f) e=f$. For $a \in \mathbf{W}$ we find

$$
\begin{aligned}
a^{x(f) x(e)} & =(a+[f, a]+(a \cdot f) f)^{x(e)} \\
& =a^{x(e)}+\left[f^{x(e)}, a^{x(e)}\right]+(a \cdot f) f^{x(e)} \\
& =a+[e, a]+(a \cdot e) e+[f, a]+[f,[e, a]]+(a \cdot e)[e, f]+(a \cdot f) f \\
& =a+[e, a]+[f, a]+[[a, e], f]+(a \cdot e) e+(a \cdot f) f,
\end{aligned}
$$

and then

$$
a^{x(f) x(e) x(e * f)}=a^{x(f) x(e)}+\left[a^{x(f) x(e)}, e * f\right] .
$$

Using Lemma 2.7 to discard most of the resulting terms, we find

$$
\left[a^{x(f) x(e)}, e * f\right]=[a, e * f]
$$

and we finally obtain

$$
\begin{aligned}
a^{x(f) x(e) x(e * f)} & =a+[e, a]+[f, a]+(a \cdot e) f+(a \cdot f) e+(a \cdot e) e+(a \cdot f) f \\
& =a+[e+f, a]+(e+f, a) \cdot(e+f)=a^{x(e+f)} .
\end{aligned}
$$

Rearranging terms yields (66). 







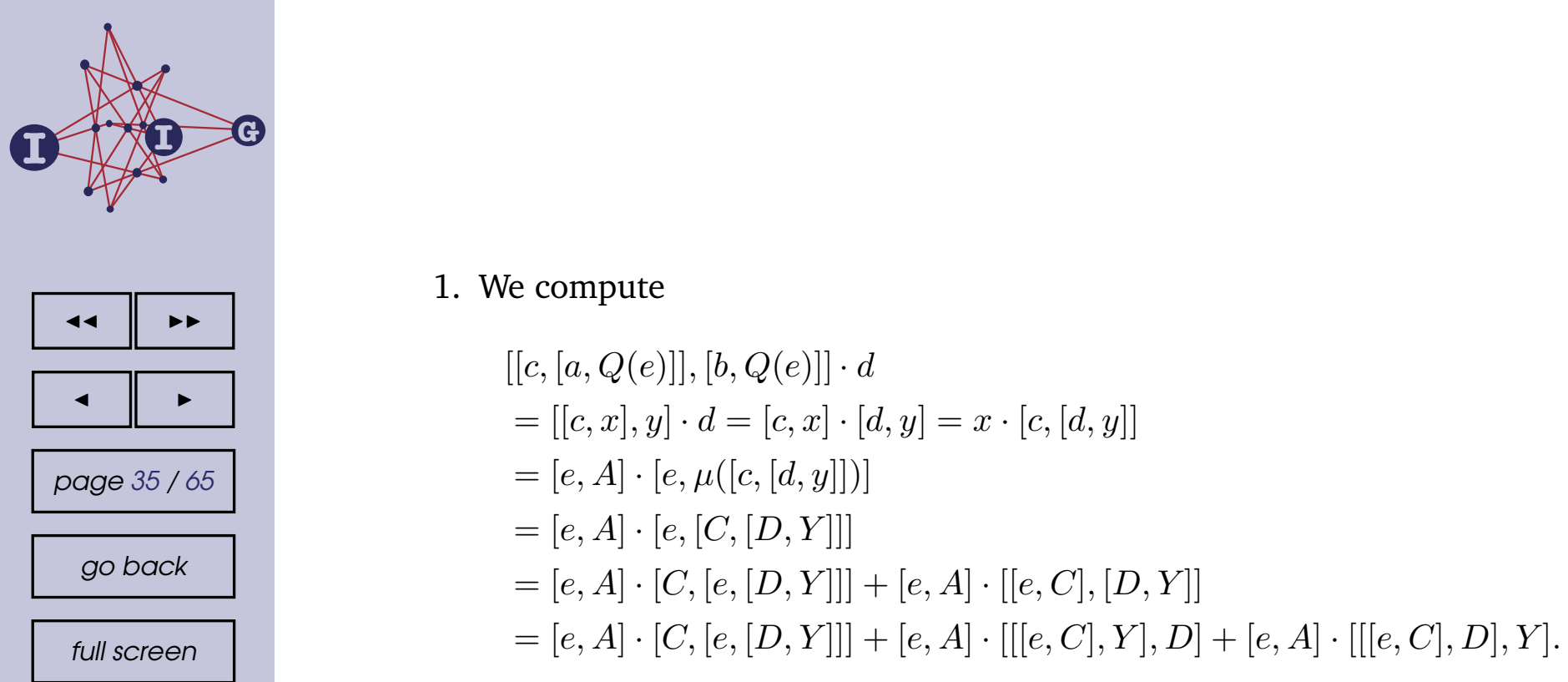

Now,

$$
\begin{aligned}
& {[e, A] \cdot[C,[e,[D, Y]]]=[e, A] \cdot\left[C, E_{b d} e\right]=E_{a c} E_{b d}} \\
& {[e, A] \cdot[[[e, C], Y], D]=[e, A] \cdot\left[E_{b c} e, D\right]=E_{b c} E_{a d}} \\
& {[e, A] \cdot[[[e, C], D], Y]=[[e, A], Y] \cdot[[e, C], D]=E_{a b} e \cdot[[e, C], D]=E_{a b} E_{c d}}
\end{aligned}
$$

This proves

$$
[[c, x], y] \cdot d=E_{a c} E_{b d}+E_{b c} E_{a d}+E_{a b} E_{c d} .
$$

Adding to this expression the expression obtained by interchanging the roles of $a$ and $b$ (and hence those of $x$ and $y$ ) yields $[c,[x, y]] \cdot d=0$, for all $c, d \in \mathbf{W}$. Hence $[x, y]=0$.

Setting $a=b$ in (81) we get $\left[c, x^{2}\right] \cdot d=0$, for all $c, d \in \mathbf{W}$. Therefore $x^{2}=0$ and by symmetry $y^{2}=0$.

Also

$$
x \cdot y=[a, Q(e)] \cdot y=[e, A] \cdot[e, Y]=e \cdot[[e, A], Y]=E_{a b} e \cdot e=0 .
$$

This proves that $x * y$ is well-defined. We find

$$
\begin{aligned}
{[c, x * y] \cdot d } & =[[c, x], y] \cdot d+(c \cdot y)(x \cdot d)+(c \cdot x)(y \cdot d) \\
& =[[c, x], y] \cdot d+E_{b c} E_{a d}+E_{a c} E_{b d},
\end{aligned}
$$

and by (81) we find that this is equal to $E_{a b} E_{c d}=E_{a b}[c, Q(e)] \cdot d$, for all $c, d \in \mathbf{W}$. Hence $x * y=([a, Q(e)] \cdot b) Q(e)$. If $Q(e) \neq 0$ we may always find $a, b$ such that $[a, Q(e)] \cdot b=1$, and then $Q(e)=x * y$. This proves that $Q(e)$ is totally isotropic with defining pair $\{x, y\}$.

2. Let $e \cdot f=0$. By Lemma 3.1-2 we have $[e, f]^{2}=0$ and therefore $[e, f]$ is 

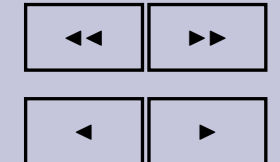

page $36 / 65$

go back

full screen

close

quit

isotropic and $Q([e, f])$ is well-defined. We compute

$$
\begin{aligned}
& {[[a, E], F] \cdot b=[x, Q(f)] \cdot b=[f, X] \cdot[f, B]} \\
& =[f, e *[e, A]] \cdot[f, B] \\
& =[[e, f],[e, A]] \cdot[f, B]+([e, A] \cdot f)(e \cdot[f, B]) \\
& =[[f, B],[e, A]] \cdot[e, f]+([e, A] \cdot f)(e \cdot[f, B]) \\
& =[[[f, B], e], A] \cdot[e, f]+[[[f, B], A], e] \cdot[e, f]+([e, A] \cdot f)(e \cdot[f, B]) \\
& =[[[f, B], e], A] \cdot[e, f]+[[f, B], A] \cdot[[e, f], e]+([e, A] \cdot f)(e \cdot[f, B]) \\
& =[[[f, B], e], A] \cdot[e, f]+([e, A] \cdot f)(e \cdot[f, B])
\end{aligned}
$$

and hence, adding the same formula with the roles of $e$ and $f$ interchanged,

$$
\begin{aligned}
{[a,[E, F]] } & =[[[f, B], e], A] \cdot[e, f]+[[[e, B], f], A] \cdot[e, f] \\
& =[[[e, f], A], B] \cdot[e, f] \\
& =[[e, f], A] \cdot[[e, f], B]=[a, Q([e, f]] \cdot b .
\end{aligned}
$$

This is true for all $a, b \in \mathbf{W}$, hence $[Q(e), Q(f)]=Q([e, f])$.

3. Note that $e+f$ is isotropic and hence $Q(e+f)$ is well-defined. Let $a, b \in \mathbf{W}$. Applying the definition of $Q(\cdot)$, we find

$$
\begin{aligned}
{[a, Q} & (e+f)] \cdot b-[a, Q(e)] \cdot b-[a, Q(f)] \cdot b \\
& =[e+f, A] \cdot[e+f, B]-[e, A] \cdot[e, B]-[f, A] \cdot[f, B] \\
& =[e, A] \cdot[f, B]+[e, B] \cdot[f, A] \\
& =[[f, B], A] \cdot e+e \cdot[[B,[f, A]] \\
& =e \cdot[f,[A, B]]=\langle e * f,[A, B]\rangle \\
& =\mu^{-1}(e * f) \cdot[a, b]=\left[a, \mu^{-1}(e * f)\right] \cdot b .
\end{aligned}
$$

This is true for all $a, b \in \mathbf{W}$, hence $Q(e+f)-Q(e)-Q(f)=\mu^{-1}(e * f)$.

4. Let $c, d \in \mathbf{W}$. Then

$$
[c, Q([e, A])] \cdot d=[[e, A], C] \cdot[[e, A], D]
$$

Also

$$
\begin{aligned}
{[c, a *[a, Q(e)]] \cdot d } & =[c, a * x] \cdot d \\
& =[c, a] \cdot[x, d]+(c \cdot x)(a \cdot d)+(c \cdot a)(x \cdot d) \\
& =[[a, c], d] \cdot x+E_{a c} a \cdot d+E_{a d} a \cdot c
\end{aligned}
$$




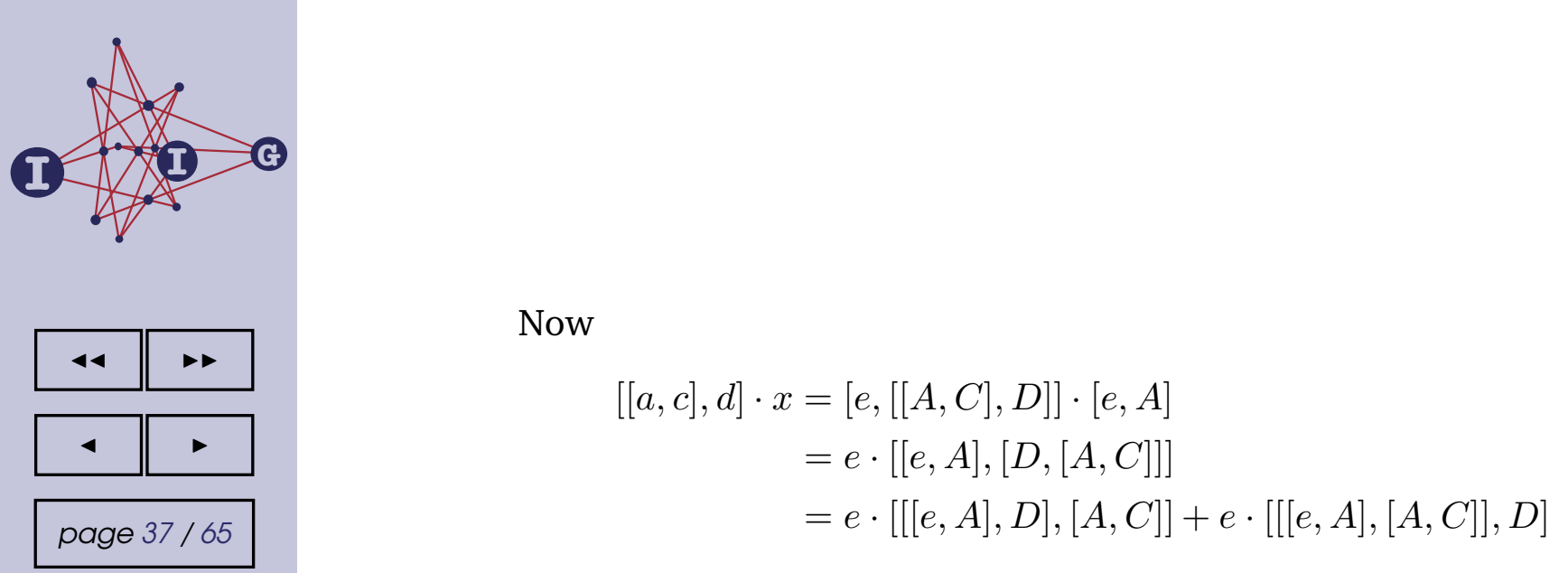

Because $A$ is totally isotropic, we may apply Lemma 3.3-2 to reduce the second term of this result to

$$
\begin{aligned}
e \cdot[[[e, A],[A, C]], D] & =e \cdot\langle A, C\rangle[[e, A], D] \\
& =(a \cdot c)[e, D] \cdot[e, A]=E_{a d} a \cdot c .
\end{aligned}
$$

The first term in the result of (83) can be simplified as follows :

$$
\begin{aligned}
e \cdot[[[e, A], D],[A, C]] & =[e,[A, C]] \cdot[[e, A], D] \\
& =[[e, C], A] \cdot[[e, A], D]+[[e, A], C] \cdot[[e, A], D] \\
& =[e, C] \cdot[[[e, A], D], A]+[[e, A], C] \cdot[[e, A], D] \\
& =E_{a c} a \cdot d+[[e, A], C] \cdot[[e, A], D]
\end{aligned}
$$

Adding (84) yields the same result as (82).

Lemma 6.3. Let e be an isotropic element of $\mathbf{W}$. Then the coordinates of $Q=Q(e)$ are given by

$$
\begin{array}{ll}
Q[t]=e[\bar{t}]^{2}, \text { when } t \in \Phi_{L}, & Q[t]=\sum_{\substack{\{u, v\} \subset \Phi_{S} \\
u+v=\bar{t}}} e[u] e[v], \text { when } t \in \Phi_{S}, \\
Q[1]=e[4]^{2}, & Q[3]=\sum_{\substack{u \in \Phi_{S} \\
\left\langle r_{1}, u\right\rangle=-1}} e[u] e[-u], \\
Q[2]=e[3]^{2}, & Q[4]=\sum_{\substack{u \in \Phi_{S} \\
\left\langle r_{2}, u\right\rangle=-1}} e[u] e[-u] .
\end{array}
$$

Proof. Let $r, s \in \Phi_{S}$. We have

$$
\left[e, e_{\bar{r}}\right]=\sum_{\substack{u \in \Phi_{S} \\\langle\bar{r}, u\rangle=-1}} e[u] e_{\bar{r}+u}, \quad\left[e, e_{\bar{s}}\right]=\sum_{\substack{v \in \Phi_{S} \\\langle\bar{s}, v\rangle=-1}} e[v] e_{\bar{s}+v},
$$

and hence, by (79),

$$
\left[e_{r}, Q(e)\right] \cdot e_{s}=\left[e, e_{\bar{r}}\right] \cdot\left[e, e_{\bar{s}}\right]=\sum_{u, v}^{\prime} e[u] e[v]
$$





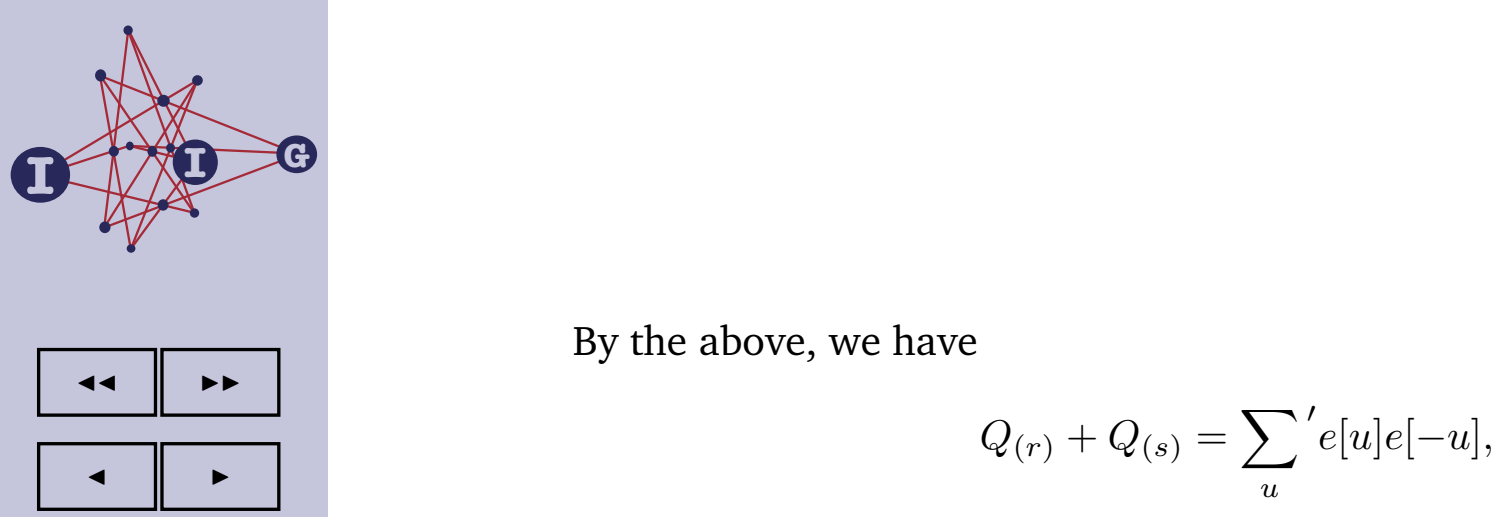

where the sum ranges over all $\{u,-u\}$ such that either $\langle\bar{r}, \pm u\rangle= \pm 1$ or $\langle\bar{s}, \pm u\rangle= \pm 1$, but not both. Note that $\bar{r}+\bar{s}=2 \bar{t}$, and hence $2\langle\bar{t}, u\rangle=$ $2\langle u, \bar{t}\rangle=\langle u, \bar{r}\rangle+\langle u, \bar{s}\rangle$, and therefore $\langle\bar{t}, u\rangle=\langle\bar{r}, u\rangle+\langle\bar{s}, u\rangle$. It follows that the sum ranges exactly over all $\{u,-u\}$ for which $\langle\bar{t}, \pm u\rangle= \pm 1$. Hence

$$
Q_{(r)}+Q_{(s)}=\sum_{\substack{u \in \Phi_{S} \\\langle u, \bar{t}\rangle=-1}} e[u] e[-u]=\sum_{\{u,-u\} \subset \Phi_{S}}\langle u, \bar{t}\rangle e[u] e[-u]
$$

Now, $e^{2}=0$ and in particular $e^{2}[3]=e^{2}[4]=0$. Using (14) this translates to

$$
0=e[3]^{2}+\sum_{\{u,-u\} \subset \Phi_{S}}\left\langle u, r_{4}\right\rangle e[u] e[-u],
$$

and similarly for $e[4]^{2}$.

Comparing this result with (86) applied to $t=r_{1}$ and $t=r_{2}$, we obtain the formulae for $Q[1]$ and $Q[2]$.

Let $r, s \in \Phi_{s}$. We may use (85) to compute the following example values of $Q(e)$ :

$$
\begin{array}{llrl}
Q\left(e_{r}\right) & =e_{\bar{r}}, & \\
Q\left(e_{r}+e_{s}\right) & =e_{\bar{r}}+e_{\bar{s}}, & \text { when }\langle r, s\rangle=1, \\
Q\left(e_{r}+e_{s}\right) & =e_{\bar{r}}+e_{\bar{s}}+e_{\overline{r+s}}, & \text { when }\langle r, s\rangle=0, \\
Q\left(e_{r}+e_{-r}+h_{r}\right) & =e_{\bar{r}}+e_{-\bar{r}}+h_{\bar{r}}, &
\end{array}
$$

We extend the Frobenius morphism $k \mapsto k^{\text {frob }} \stackrel{\text { def }}{=} k^{2}$ over $K$ to $\mathbf{F}$ by applying it to the coordinates with respect to the Chevalley basis. When $K$ is a perfect field this map is nonsingular and then ${ }^{\text {frob }}$ is a field automorphism of $\mathbf{F}$.

The three left hand equations of (85) imply

$$
Q(e)=\mu\left(e^{\text {frob }}\right) \bmod \mathbf{W}, \quad \text { for isotropic } e \in \mathbf{W} .
$$

Proposition 6.4. Let $e, f \in \mathbf{W}$ be such that $e^{2}=f^{2}=0$. Then

1. $\langle Q(e), Q(f)\rangle=(e \cdot f)^{2}$.

2. $Q([e, Q(f)])=f^{\text {frob }} *\left[Q(e), f^{\text {frob }}\right]$. 


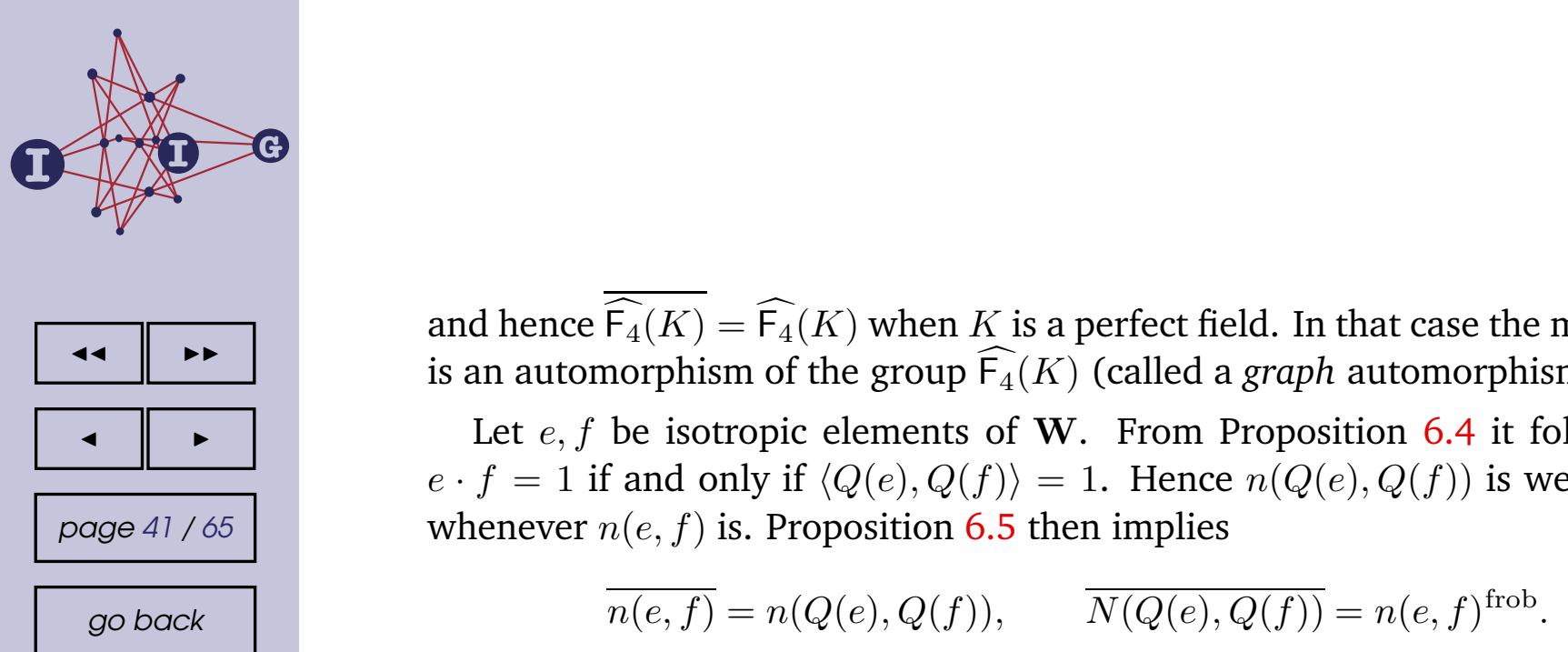

In particular

full screen

close

and then

$$
\overline{n_{r}(k)}= \begin{cases}n_{\bar{r}}\left(k^{2}\right), & \text { when } r \in \Phi_{S}, \\ n_{\bar{r}}(k), & \text { when } r \in \Phi_{L},\end{cases}
$$

$$
\overline{h_{r}(k)}= \begin{cases}h_{\bar{r}}\left(k^{2}\right), & \text { when } r \in \Phi_{S}, \\ h_{\bar{r}}(k), & \text { when } r \in \Phi_{L} .\end{cases}
$$

Using Lemma 5.1 the case $k=1$ of (92) can easily be generalized to

$$
\overline{n(w)}=n(\bar{w}), \quad \text { for all } w \in W .
$$

Proposition 6.6. Let $e$ be an isotropic element of $\mathbf{W}$. Let $g$ be an automorphism of $\mathbf{W}$ (and $\mathbf{F}$ ). Then

$$
Q\left(e^{g}\right)=Q(e)^{\bar{g}}
$$

Proof. Let $a, b \in \mathbf{W}, g \in \widehat{\mathrm{F}_{4}}(K)$. We have

$$
\begin{aligned}
{\left[a^{\bar{g}}, Q(e)^{\bar{g}}\right] \cdot b^{\bar{g}} } & =[a, Q(e)] \cdot b \\
& =[e, \mu(a)] \cdot[e, \mu(b)] \\
& =\left[e^{g}, \mu(a)^{g}\right] \cdot\left[e^{g}, \mu(b)^{g}\right] \\
& =\left[e^{g}, \mu\left(a^{\bar{g}}\right)\right] \cdot\left[e^{g}, \mu\left(b^{\bar{g}}\right)\right]=\left[a^{\bar{g}}, Q\left(e^{g}\right)\right] \cdot b^{\bar{g}}
\end{aligned}
$$

\section{Octagonality}

From now on we shall require that $K$ has a Tits automorphism, i.e., a field automorphism $\sigma$ with the property

$$
\left(k^{\sigma}\right)^{\sigma}=k^{2}, \quad \text { for every } k \in K \text {. }
$$

Note that we require $\sigma$ to have an inverse $\sigma^{-1}$, and consequently, that $K$ is a perfect field. 
For finite fields a Tits automorphism exists if and only if the order of the field is an odd power $2^{2 m+1}$ of 2 , and then $k^{\sigma} \stackrel{\text { def }}{=} k^{2^{m+1}}$.

As with the Frobenius automorphism, we shall extend $\sigma$ to $\mathbf{F}$ by setting $e_{r}^{\sigma} \stackrel{\text { def }}{=}$ $e_{r}, h_{r}^{\sigma} \stackrel{\text { def }}{=} h_{r}$, for $r \in \Phi$. We have $A^{\text {frob }}=\left(A^{\sigma}\right)^{\sigma}$ for every $A \in \mathbf{F}$. As before, when $g$ is an automorphism of $\mathbf{W}$, we shall write $g^{\sigma}=\sigma^{-1} g \sigma$. Note that $\bar{\sigma}=\sigma$ and hence $\overline{g^{\sigma}}=\bar{g}^{\sigma}$. By Lemma 4.5 we have $x(e)^{\sigma}=x\left(e^{\sigma}\right)$ and $x(E)^{\sigma}=x\left(E^{\sigma}\right)$, when $e$ (resp. $E$ ) is isotropic (resp. totally isotropic).

For $a, e \in \mathbf{W}$ such that $e$ is isotropic we define the operator $q(\cdot, \cdot)$ as follows :

$$
q(a, e) \stackrel{\text { def }}{=}\left[a, Q\left(e^{\sigma^{-1}}\right)\right] .
$$

We write $q(\mathbf{W}, e)$ for the set of elements $q(a, e)$ with $a \in \mathbf{W}$, i.e., $q(\mathbf{W}, e)=$ $\left[\mathbf{W}, Q\left(e^{\sigma^{-1}}\right)\right]$.

(This is the last operator which we shall need to introduce in this text. It serves an important purpose in the definition of the Ree group and the ReeTits octagon and, as shall be shown in Theorem 10.1, also has an interesting geometric interpretation.)

An element $e \in \mathbf{W}$ shall be called semi-octagonal if and only if it is isotropic and $q(e, e)=0$. An element $e \in \mathbf{W}$ shall be called octagonal if and only if it is isotropic and $e \in q(\mathbf{W}, e)$, or equivalently (by Lemma 4.8) if and only if $q([e, \mathbf{W}], e)=0$. All octagonal elements are semi-octagonal, but not conversely.

Proposition 7.1. Let $e, f$ be isotropic elements of $\mathbf{W}$. Then

1. $Q\left(q(e, f)^{\sigma^{-1}}\right)=f * q(f, e)$,

2. $q(e, f)=0$ if and only if $q(f, e)=0$,

3. $e \in q(\mathbf{W}, f)$ if and only if $f \in q(\mathbf{W}, e)$,

4. $q(e, e)$ is octagonal and $e \in q(\mathbf{W}, q(e, e))$.

Proof. Statements 1-3 are immediate consequences of Proposition 6.4 with $f^{\sigma^{-1}}$ substituted for $f$. Setting $f=e$ in the first statement of this proposition, we see that $\{e, q(e, e)\}$ is a defining pair for $Q\left(q(e, e)^{\sigma^{-1}}\right)$. Hence both $e$ and $q(e, e)$ belong to $\left[\mathbf{W}, Q\left(q(e, e)^{\sigma^{-1}}\right)\right]$ and therefore $q(e, e)$ is octagonal.

To study the (semi-) octagonal elements of $\mathbf{W}$, we first look at elements of the form $e_{r}$ with $r \in \mathbf{W}$. Table 1 lists the 24 short roots $r \in \Phi_{S}$, the corresponding long roots $\bar{r}$ and the value of $r+\bar{r}$ when it belongs to $\Phi_{S}$. 


$$
\langle r, \bar{r}\rangle=1
$$

\begin{tabular}{|c|c|}
\hline$r$ & $\bar{r}$ \\
\hline 0100 & 0110 \\
$0 \overline{1} 00$ & $0 \overline{1} \overline{1} 0$ \\
0001 & 1001 \\
$000 \overline{1}$ & $\overline{1} 00 \overline{1}$ \\
++++ & 0101 \\
+--+ & $0 \overline{1} 01$ \\
-++- & $010 \overline{1}$ \\
---- & $0 \overline{1} 0 \overline{1}$ \\
\hline
\end{tabular}

$$
\langle r, \bar{r}\rangle=0
$$

\begin{tabular}{|c|c|}
\hline$r$ & $\bar{r}$ \\
\hline+++- & $\overline{1} 100$ \\
++-+ & 0011 \\
+-++ & $00 \overline{1} 1$ \\
+--- & $\overline{1} \overline{1} 00$ \\
-+++ & 1100 \\
-+-- & $001 \overline{1}$ \\
--+- & $00 \overline{1} \overline{1}$ \\
---+ & $1 \overline{1} 00$ \\
\hline
\end{tabular}

$\langle r, \bar{r}\rangle=-1$

\begin{tabular}{|c|c||c|}
\hline$r$ & $\bar{r}$ & $r+\bar{r}$ \\
\hline 1000 & $\overline{1} 001$ & 0001 \\
$\overline{1} 000$ & $100 \overline{1}$ & $000 \overline{1}$ \\
0010 & $01 \overline{1} 0$ & 0100 \\
$00 \overline{1} 0$ & $0 \overline{1} 10$ & $0 \overline{1} 00$ \\
++-- & $\overline{1} 010$ & -++- \\
+-+- & $\overline{1} 0 \overline{1} 0$ & ---- \\
-+-+ & 1010 & ++++ \\
--++ & $10 \overline{1} 0$ & +--+ \\
\hline
\end{tabular}

Table 1: Short roots $r \in \Phi_{S}$ and the corresponding long roots $\bar{r}$.

The leftmost list corresponds to octagonal elements $e_{r}$, the middle list corresponds to those $e_{r}$ that are semi-octagonal but not octagonal and for the rightmost list elements are not even semi-octagonal. In the last case the third column corresponds to the value of $q\left(e_{r}, e_{r}\right)$.

Lemma 7.2. Consider Weyl group elements $w, w^{\prime}$ of the form $w=w_{r} w_{\bar{r}}$, where $r \in \Phi_{S}$ such that $\langle r, \bar{r}\rangle=0$, and $w^{\prime}=\left(w_{s} w_{\bar{s}}\right)^{2}$ with $s \in \Phi_{S}$ such that $\langle s, \bar{s}\rangle=1$.

Then $\bar{w}=w$ and $\bar{w}^{\prime}=w^{\prime}$. The elements of this form generate a subgroup $W^{\prime}$ of $W$ which is isomorphic to the dihedral group of order 16. This group has 3 orbits on $\Phi_{S}$ which correspond to the three lists of 8 roots given in Table 1.

Proof. When $\langle r, \bar{r}\rangle=0$ the Weyl group elements $w_{r}$ and $w_{\bar{r}}$ commute. Then $\bar{w}=\overline{w_{r} w_{\bar{r}}}=\overline{w_{r}} \overline{w_{\bar{r}}}=w_{\bar{r}} w_{r}=w$.

When $\langle s, \bar{s}\rangle=1$ we have $w^{\prime}=\left(w_{s} w_{\bar{s}}\right)^{2}=w_{\bar{s}}^{w_{s}} w_{\bar{s}}=w_{w_{s}(\bar{s})} w_{\bar{s}}=w_{s-\bar{s}} w_{\bar{s}}$. Also $\overline{s-\bar{s}}=\bar{s}-2 s$, hence $\overline{w^{\prime}}=w_{\bar{s}-2 s} w_{s}=w_{s} w_{s} w_{\bar{s}-2 s} w_{s}=w_{s} w_{\bar{s}-2 s}^{w_{s}}=w_{s} w_{\bar{s}-2 s+s}=$ $w_{s} w_{\bar{s}-s}$. But also $w^{\prime}=\left(w_{s} w_{\bar{s}}\right)^{2}=w_{s} w_{s}^{w_{\bar{s}}}=w_{s} w_{s-\bar{s}}$.

For example, with $s=0100$ we obtain $w^{\prime}$ which maps roots $(x, y, z, t)$ to $(x,-y,-z, t)$. With $r=+++-$ we obtain $w$, which maps $(x, y, z, t)$ to $\frac{1}{2}(-x+y-$ $z+t, x-y-z+t,-x-y+z+t, x+y+z+t)$. We leave it to the reader to compute $W^{\prime}$ and prove that it has the stated properties.

\section{The Ree group ${ }^{2} \widehat{\mathrm{F}_{4}}(\boldsymbol{K})$}

Proposition 8.1. Let $g$ be an automorphism of $\mathbf{W}$ satisfying $\bar{g}=g^{\sigma}$, i.e.,

$$
\left(a^{g}\right)^{\sigma}=\left(a^{\sigma}\right)^{\bar{g}}, \quad \text { for all } a \in \mathbf{W} .
$$





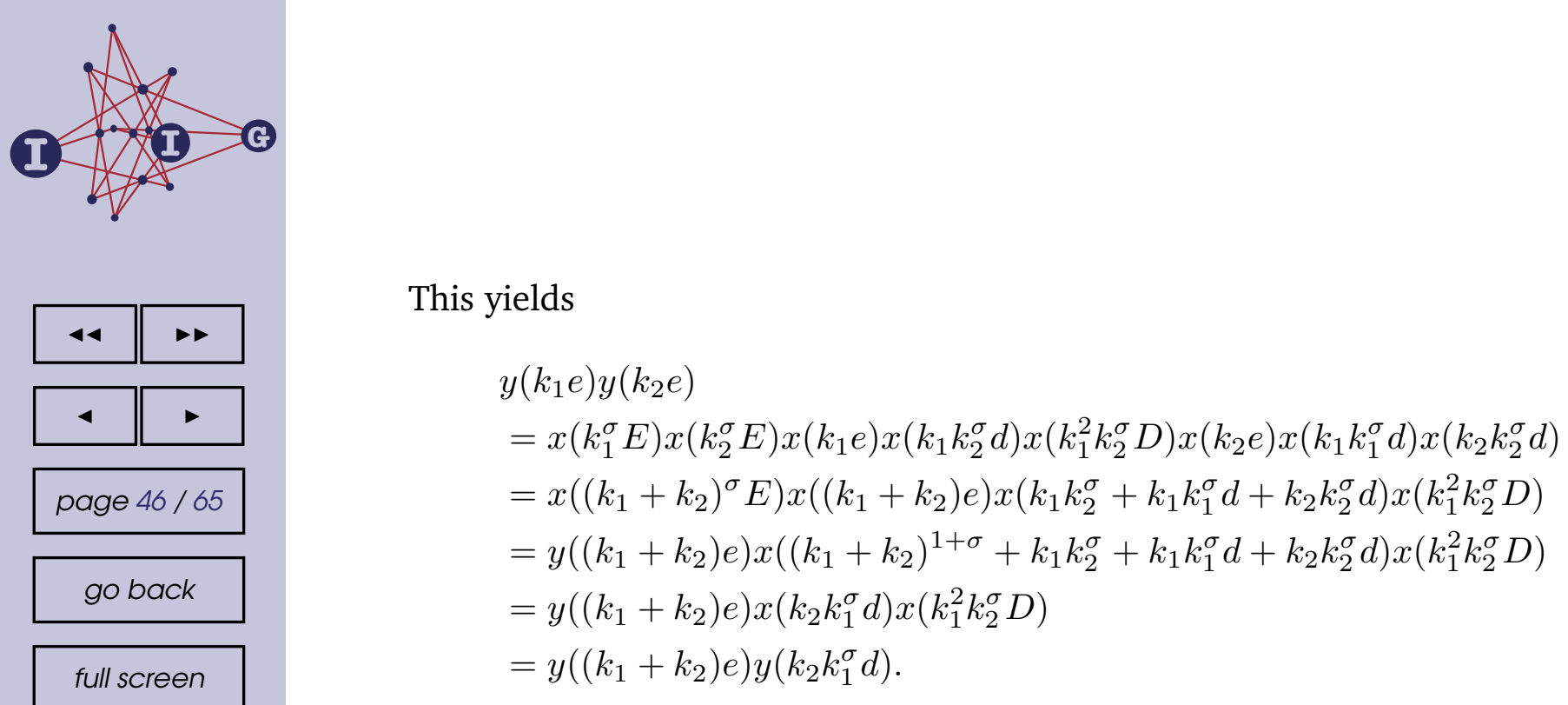

where we use Propositions 4.3 and 4.4 to simplify the products of the form $x(k e) x\left(k^{\prime} e\right)$ and $x(k E) x\left(k^{\prime} E\right)$.

Lemma 8.4. Let $e$ be an octagonal (isotropic) element of $\mathbf{W}$. Let $g \in{ }^{2} \widehat{\mathrm{F}_{4}}(K)$. Then

$$
y\left(e^{g}\right)=y(e)^{g} .
$$

Proof. We have $y\left(e^{g}\right)=x\left(Q\left(e^{g}\right)^{\sigma^{-1}}\right) x\left(e^{g}\right)$. Using (98) we obtain $Q\left(e^{g}\right)^{\sigma^{-1}}=$ $Q(e)^{\bar{g} \sigma^{-1}}=Q(e)^{\sigma^{-1} g}$ and hence $y\left(e^{g}\right)=x\left(Q\left(e^{\sigma^{-1}}\right)^{g}\right) x\left(e^{g}\right)$, which by (64) is equal to $x\left(Q\left(e^{\sigma^{-1}}\right)\right)^{g} x(e)^{g}=y(e)^{g}$.

The remainder of this section shall be devoted to show transitivity of ${ }^{2} \widehat{\mathrm{F}_{4}}(K)$ on the set of octagonal elements of $\mathbf{W}$. In order to do this we shall make use of a 'reduction process' which can be summarized as follows : we choose an element of the form $y_{t}(k)$ to map a given $a \in \mathbf{W}$ onto $a^{\prime} \in \mathbf{W}$ with the property that $a^{\prime}$ has one more coordinate that is 'known' to be zero. Successive reductions reduce the number of non-zero coordinates of $a$ while staying in the same orbit of $\widehat{\mathrm{F}_{4}}(K)$, until the final image of $a$ has a sufficiently simple structure.

In general, when it is known that $a[r] \neq 0$ for some $r \in \Phi_{S}$, we choose $s \in \Phi_{S}$ such that $\langle r, s\rangle=1$ and then apply an element of the form $y_{t}(k)$ with $t=s-r$. From (63) and (102) it follows that the coordinate of the image $a^{\prime}$ of $a$ at position $s$ is now equal to $a^{\prime}[s]=a[s]+k a[r]$. Hence, setting $k=a[s] / a[r]$ we obtain $a^{\prime}[s]=0$. When $\langle r, s\rangle=0$ we obtain a similar effect when we use $t$ such that $\bar{t}=s-r$. Now $a^{\prime}[s]=a[s]+k^{\sigma} a[r]$ and we take $k=(a[s] / a[r])^{\sigma^{-1}}$.

This transformation does however also affect other coordinates $a[s]$ of $a$, and when performing successive reductions, we should make sure that earlier annihilations are not 'undone' by later actions.

Consider how the action of $x_{r}(k)$ affects coordinates $a[s]$ of $a$, with $s \in \Phi_{S}$. (Coordinates $a[3]$ and $a[4]$ will turn out not to be important). It follows from (63) that only those coordinates are affected that satisfy $\langle r, s\rangle>0$. As a consequence, the action of $y_{r}(k)$ will only affect those coordinates for which $\langle r, s\rangle>0$ 



From $[e, f]=\left[e_{----}, f\right] \neq 0$ we easily deduce that either $f[3]+f[4] \neq 0$ or at least one of the coordinates $f[r] \neq 0$, with $r=1000,0100,0010,0001,-+++$, ,+-++++-+ or +++- .

Below we give a list of group elements $y_{t}(1)$ which can be used to map $f$ onto $f^{\prime}$ with the required properties, whenever $f[r] \neq 0$ for one of the given $r$

\begin{tabular}{|c|c|ccc|}
\hline$r$ & $s$ & $\bar{t}$ & $t$ & $\bar{t}+t$ \\
\hline 1000 & +++- & $010 \overline{1}$ & -++- & \\
0100 & & \multicolumn{3}{|c|}{ none needed } \\
0010 & +++- & $\overline{1} 010$ & ++-- & -++- \\
0001 & 0100 & $010 \overline{1}$ & -++- & \\
-+++ & 0100 & $\overline{1} 010$ & ++-- & -++- \\
+-++ & +++- & $010 \overline{1}$ & -++- & \\
++-+ & 0100 & $010 \overline{1}$ & -++- & \\
+++- & 0100 & $10 \overline{1} 0$ & --++ & \\
\hline
\end{tabular}

For example, the last row of the table tells us that, when $f[0100]=0$ but $f[+++-] \neq 0$, we may apply $y_{--++}(1)$ to create a non-zero coordinate at position 0100. Similarly, the first row of the table indicates that when $f[0100]=$ $f[+++-]=0$ but $f[1000] \neq 0$ we may first apply $y_{-++-}(1)$ to obtain an image with a non-zero coordinate at position +++-, bringing us back to the first example. We leave it to the reader to verify that each of the listed group elements indeed leaves $e=e$ invariant.

Only the case where $f[r]=0$ for each $r$ in the table, remains. It turns out that now $f^{2}=0$ implies $f[3]+f[4]=0$, contradicting $[e, f]=0$. Indeed, we may use (14) to compute $f^{2}[3]+f^{2}[4]=f[3]^{2}+f[4]^{2}=(f[3]+f[4])^{2}$.

Henceforth we may assume that the coordinate $f[0100]$ is non-zero. We can map $f$ onto $k e_{0100}$ using the reductions $y_{t}(k)$ of Table 2 , except the first reduction (which corresponds to $s=++++$ ). We have no need for this reduction because $e \cdot f=e_{----} \cdot f=0$ implies $f[++++]=0$. It is easily verified that all other reductions in the table satisfy $\langle t,----\rangle \geq 0$ and $\langle\bar{t},----\rangle \geq 0$ and therefore leave $e$ invariant.

This proves that $f$ can be mapped onto an element of the form $k e_{0100}$. We get rid of the scalar $k$ by applying $i_{s}(k)$ with $s=--++$ and $\bar{s}=10 \overline{1} 0$. Indeed $\langle--++, 0100\rangle=-1, \quad\langle 10 \overline{1} 0,0100\rangle=0, \quad\langle--++,----\rangle=0, \quad\langle 10 \overline{1} 0,----\rangle=0$, and hence $i_{s}(k)$ leaves $e$ invariant and maps $k e_{0100}$ to $e_{0100}$. 



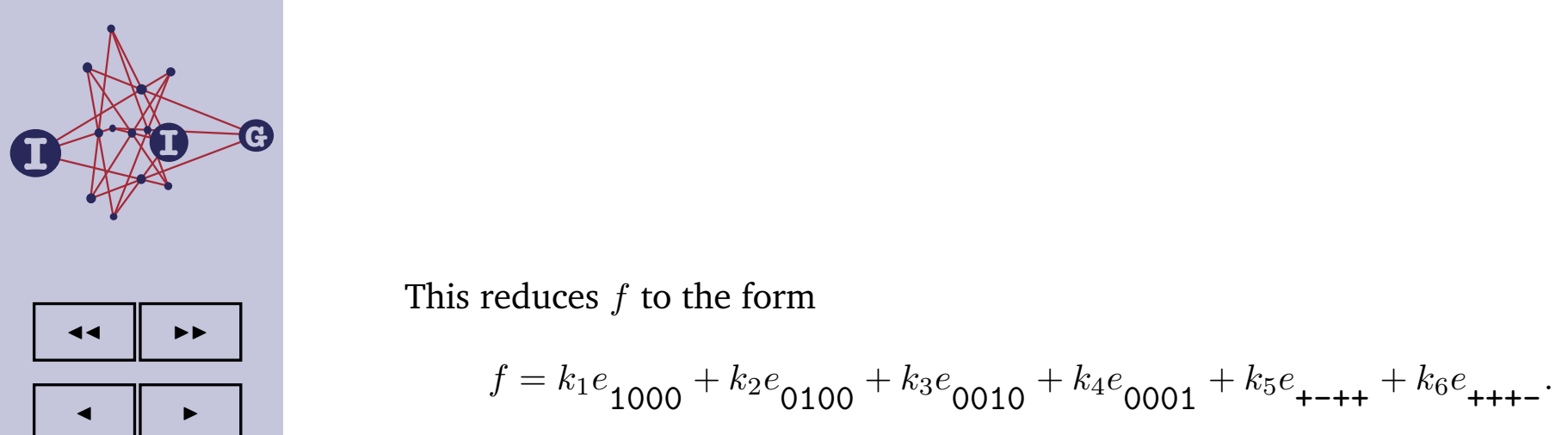

We compute

page 54 / 65

go back

full screen

close

quit

$$
\begin{array}{r}
Q(f)=k_{1}^{2} e_{\overline{1} 001}+k_{2}^{2} e_{0110}+k_{3}^{2} e_{01 \overline{1} 0}+k_{4}^{2} e_{1001}+k_{5}^{2} e_{00 \overline{1} 1}+k_{6}^{2} e_{\overline{1} 100} \\
+k_{1} k_{2} e_{-+++}+k_{1} k_{4} e_{0001}+k_{2} k_{3} e_{0100}+k_{2} k_{4} e_{++++}+k_{3} k_{4} e_{++-+} \\
+\left(k_{1} k_{3}+k_{5} k_{6}\right) e_{-+-+}
\end{array}
$$

Now, as $f$ is octagonal, we have $0=\left[\left[f^{\sigma}, a\right], Q(f)\right] \cdot b=\left[f^{\sigma}, a\right] \cdot[Q(f), b]$ for all $a, b \in \mathbf{W}$. Setting $a=e_{--+-}$and $b=e_{++--}$we obtain

$$
\begin{gathered}
{\left[f^{\sigma}, e_{--+-}\right]=k_{1}^{\sigma} e_{+-+-}+k_{2}^{\sigma} e_{-++-}+k_{4}^{\sigma} e_{--++}} \\
{\left[Q(f), e_{++--}\right]=k_{1}^{2} e_{-+-+}+k_{1} k_{2} e_{0100}+k_{1} k_{4} e_{++-+}}
\end{gathered}
$$

and therefore $\left[f^{\sigma}, e_{--+-}\right] \cdot\left[Q(f), e_{++--}\right]=k_{1}^{\sigma} k_{1}^{2}$.

Likewise,

$$
\begin{aligned}
& {\left[f^{\sigma}, e_{+---}\right] \cdot\left[Q(f), e_{--++}\right]=k_{3}^{\sigma} k_{3}^{2},} \\
& {\left[f^{\sigma}, e_{-++-}\right] \cdot\left[Q(f), e_{000 \overline{1}}\right]=k_{5}^{\sigma} k_{5}^{2},} \\
& {\left[f^{\sigma}, e_{+--+}\right] \cdot\left[Q(f), e_{0 \overline{1} 00}\right]=k_{6}^{\sigma} k_{6}^{2},}
\end{aligned}
$$

proving that $k_{1}=k_{3}=k_{5}=k_{6}=0$ when $f$ is octagonal. Hence we are left with $f=k_{2} e_{0100}+k_{4} e_{0001}$, with $k_{2} \neq 0$. In the proof of Theorem 8.5 (Stage 2) it was already shown that an element of this form can only be octagonal when $k_{4}=0$.

In a final step we get rid of the constant $k_{2}$ by applying $i_{s}\left(k_{2}\right)$ with $s=--++$ and $\bar{s}=10 \overline{1}$, the same element we used in the proof of Lemma 9.2.

We combine Lemmas 9.1-9.4 into the following

Theorem 9.5. The following is an exhaustive list of all orbits of ${ }^{2} \widehat{\mathrm{F}_{4}}(K)$ on ordered pairs $(e, f)$ of non-zero octagonal (isotropic) elements $e, f \in \mathbf{W}$.

1. For each $k \in K-\{0\}$ an orbit with representative $\left(e_{0100}, k e_{0 \overline{1} 00}\right)$. 


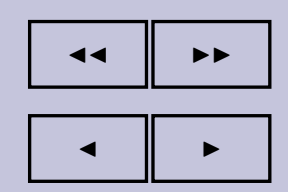

page $55 / 65$

go back

full screen

close

quit

2. An orbit with representative $\left(e_{0100}, e_{----}\right)$.

3. An orbit with representative $\left(e_{0100}, e_{0001}\right)$.

4. An orbit with representative $\left(e_{0100}, e_{++++}\right)$.

5. For each $k \in K-\{0\}$ an orbit with representative $\left(e_{0100}, k e_{0100}\right)$.

The following table lists several properties of the corresponding pairs $(e, f)$.

\begin{tabular}{|c||c|c|c|c|c|}
\hline & Case 1 & Case 2 & Case 3 & Case 4 & Case 5 \\
\hline$e \cdot f$ & $k$ & 0 & 0 & 0 & 0 \\
\hline$[e, f]$ & not isotr. & semi-oct. $\dagger$ & 0 & 0 & 0 \\
\hline$q(e, f)$ & isotr. $f$ & $\neq 0$, oct. & 0 & 0 & 0 \\
\hline$f \in[e, \mathbf{F}]$ & no & no & yes & yes & yes \\
\hline$[e, \mathbf{W}] \cdot f$ & $\neq 0$ & $\neq 0$ & 0 & 0 & 0 \\
\hline$e+f$ & not isotr. & not isotr. & semi-oct. $\dagger$ & octag. & octag. \\
\hline$\mu^{-1}(e * f)^{-1}$ & undef. & undef. & $\neq 0$, oct. & 0 & 0 \\
\hline$f \in[e, \mathbf{W}]$ & no & no & no & yes & yes \\
\hline$f \in q(\mathbf{W}, e)$ & no & no & no & yes & yes \\
\hline$[e, \mathbf{F}] \cdot f$ & $\neq 0$ & $\neq 0$ & $\neq 0$ & 0 & 0 \\
\hline$f \in K e$ & no & no & no & no & $f=k e$ \\
\hline$q(\mathbf{W}, e) \cap$ & $\{0\}$ & $\{0\}$ & $\begin{array}{c}K \mu^{-1} \\
(e * f)^{-1}\end{array}$ & dim $=3$ & $\begin{array}{c}q(\mathbf{W}, e)= \\
q(\mathbf{W}, f)\end{array}$ \\
\hline
\end{tabular}

$\dagger$ semi-octagonal, but not octagonal $\ddagger$ isotropic, but not semi-octagonal

Proof. Only the table of properties remains to be checked. Note that all properties are 'invariant' for ${ }^{2} \widehat{\mathrm{F}_{4}}(K)$, for example, when $g \in{ }^{2} \widehat{\mathrm{F}_{4}}(K)$, then $[e, f]^{g}=$ $\left[e^{g}, f^{g}\right], q(e, f)^{g}=q\left(e^{g}, f^{g}\right)$, etc. The only invariant which has not yet been proved elsewhere in this text is $\mu^{-1}(e * f)^{\sigma^{-1}}$. We find

$$
\mu^{-1}\left(e^{g} * f^{g}\right)^{\sigma^{-1}}=\mu^{-1}\left((e * f)^{g}\right)^{\sigma^{-1}}=\mu^{-1}(e * f)^{\bar{g} \sigma^{-1}}=\mu^{-1}(e * f)^{\sigma^{-1} g},
$$

using (98) and the definition of $\bar{g}$.

As a consequence it is sufficient to check all listed properties only for a single (representative) pair in each orbit. We shall discuss the most difficult cases here and leave the rest to be verified by the reader. 
Table 3 lists the relevant values for $f=e_{r}$ and $Q(f)=e_{\bar{r}}$ and the six corresponding base elements $e_{s_{1}}, \ldots, e_{s_{6}}$ of $q(\mathbf{W}, f)$. The last row corresponds to $f=e$. From this table we easily derive the results for $q(\mathbf{W}, e) \cap q(\mathbf{W}, f)$. Note

\begin{tabular}{|c|c|cccccc|}
\hline$r$ & $\bar{r}$ & $s_{1}$ & $s_{2}$ & $s_{3}$ & $s_{4}$ & $s_{5}$ & $s_{6}$ \\
\hline $0 \overline{1} 00$ & $0 \overline{1} \overline{1} 0$ & $0 \overline{1} 00$ & $00 \overline{1} 0$ & +--+ & +--- & ---+ & ---- \\
---- & $0 \overline{1} 0 \overline{1}$ & $0 \overline{1} 00$ & $000 \overline{1}$ & +-+- & +--- & --+- & ---- \\
0001 & 1001 & 1000 & 0001 & +--+ & +-++ & ++-+ & ++++ \\
++++ & 0101 & 0100 & 0001 & ++++ & ++-+ & -+++ & -+-+ \\
0100 & 0110 & 0100 & 0010 & ++++ & +++- & -+++ & -++- \\
\hline
\end{tabular}

Table 3: Auxiliary table for the proof of Theorem 9.5

that in the third case the intersection is generated by the single element $e_{++++}$. In that case $e * f=e_{0101}$ and indeed $\mu^{-1}(e * f)^{\sigma^{-1}}=e_{++++}$.

Consider the value of $e+f$. When $e=e_{0100}$ and $f=e_{0001}$ it was already shown in the proof of Theorem 8.5 (Stage 2) that $e+f$ is semi-octagonal but not octagonal. When $f=e_{\mathbf{+ +}_{++}}$we obtain $Q(e+f)=Q\left(e_{0100}+e_{++++}\right)=$ $e_{0110}+e_{0101}$, and then $\left[e_{000 \overline{1}}+e_{+--+}, Q(e+f)\right]=(e+f)^{\sigma}$, proving that $e+f$ is octagonal.

\section{The Ree-Tits octagon}

Because scalar multiples of octagonal elements are again octagonal, it is natural to define a new geometry $\mathcal{O}$ whose points are the one dimensional subspaces $K e$ of $\mathbf{F}$ (i.e., the points of the 25 -dimensional projective space associated with W) for which $e$ is octagonal (and isotropic).

Let $K e, K f \in \mathcal{O}$ be such that $K e \neq K f$. As an immediate consequence of the properties listed in Theorem 9.5, we find that the following are equivalent :

- Every 1-dimensional subspace $K\left(k_{1} e+k_{2} f\right)$ of $K e+K f$ belongs to $\mathcal{O}$.

- $f \in q(\mathbf{W}, e)$.

- $f \in[e, \mathbf{W}]$.

- $[e, \mathbf{F}] \cdot f=0$.

- $\operatorname{dim} q(\mathbf{W}, e) \cap q(\mathbf{W}, f)=3$. 


$$
\text { - } e \cdot f=0,[e, f]=0 \text { and } e * f=0 \text {. }
$$

Two different points $K e, K f$ of $\mathcal{O}$ that satisfy one of these statements shall be called collinear. In this case, the 2-dimensional subspace $K e+K f$ of $\mathcal{O}$ is called a line of $\mathcal{O}$.

We shall also introduce the following terminology, borrowed from [5]. Let $K e, K f$ be different points of $\mathcal{O}$.

- If $K e, K f$ are not collinear, and $[e, f]=0$, then $K e$ and $K f$ are said to be cohyperlinear.

- If $[e, f] \neq 0$ and $e \cdot f=0$, then $K e$ and $K f$ are said to be almost opposite.

- If $e \cdot f \neq 0$, then $K e$ and $K f$ are said to be opposite.

The next and most important theorem of this text proves that the point-line geometry $\mathcal{O}$ satisfies the axioms of a generalized octagon, where 'collinear', 'cohyperlinear', 'almost opposite' and 'opposite' correspond to distance 2, 4, 6 and 8 in the incidence graph of $\mathcal{O}$.

Theorem 10.1. Let $K e, K f$ be different points of $\mathcal{O}$.

1. If $K e$ and $K f$ are collinear then every point of the line $K e+K f$ belongs to $\mathcal{O}$. Apart from the points of $K e+K f$ there are no other points of $\mathcal{O}$ collinear to both $K e$ and $K f$.

2. If $K e$ and $K f$ are cohyperlinear, then there is a unique point of $\mathcal{O}$ that is collinear to both $K e$ and $K f$. This point is $K \mu^{-1}(e * f)^{\sigma^{-1}}$. In the incidence graph of $\mathcal{O}, K e$ and $K f$ are at mutual distance 4.

3. If $K e$ and $K f$ are almost opposite then there are no points of $\mathcal{O}$ that are collinear to both $K e$ and $K f$. There is a unique point of $\mathcal{O}$ that is collinear to $K e$ and cohyperlinear to $K f$. This point is $K q(f, e)$. In the incidence graph of $\mathcal{O}, K e$ and $K f$ are at mutual distance 6.

4. If $K e$ and $K f$ are opposite, then there are no points of $\mathcal{O}$ that are collinear to both $K e$ and $K f$, or that are collinear to Ke and cohyperlinear to $K f$ (or vice versa). Every line of $\mathcal{O}$ through $K f$ contains exactly one point of $\mathcal{O}$ that is almost opposite to Ke. In the incidence graph of $\mathcal{O}, K e$ and $K f$ are at mutual distance 8 .

These are the only possible relations between different points of $\mathcal{O}$.

Proof. The different cases of this theorem correspond to 4 different cases of Theorem 9.5, but numbered the other way round. (The fifth case of Theorem 







